\title{
Electrical Bio-impedance Proximity Sensing for Vitreo-retinal Micro-surgery*
}

\author{
L. Schoevaerdts ${ }^{1}$, G. Borghesan ${ }^{1,2}$, M. Ourak ${ }^{1}$, D. Reynaerts ${ }^{1,2}$ and E. Vander Poorten ${ }^{1}$
}

\begin{abstract}
Retinal Vein Occlusion (RVO) is a widespread eye vascular disease leading to vision loss because of clots obstructing a retinal vessel. Recent research investigated retinal vein cannulation, a promising treatment in which a microneedle injects a thrombolytic agent inside the clotted vessel. During this surgical procedure, the surgeon only relies on a microscope, looking through the patient eye's lens. Such visual feedback gives poor depth perception, with a risk to inject the agent underneath the retina. Such situation endangers the patient's eyesight and is to be avoided. This paper explores bioimpedance as a means to estimate the proximity between an insulated electrode and a retinal vessel. Experiments on 5 exvivo pig eyes showed that the impedance phase decreases as the electrode tip approaches the retinal vessel. Specific patterns in the impedance magnitude were detected. A dedicated algorithm showed $98 \%$ sensitivity and $100 \%$ specificity at a distance of $775 \mu \mathrm{m} \pm 275 \mu \mathrm{m}$ away from the retinal vessels. Knowledge of the proximity can help the surgeon prevent damaging fragile retinal structures during retinal surgeries such as cannulation and scar tissue peeling. A minimally-invasive measurement principle of this technique is demonstrated on an ex-vivo pig head to show the feasibility of such technique in more realistic conditions.
\end{abstract}

\section{INTRODUCTION}

\section{A. $R V O$ and vein cannulation}

About 16.4 millions persons are currently suffering from Retinal Vein Occlusion (RVO), making RVO the second most common retinal vascular disorder [1]. In RVO, clots obstruct retinal vessels, forcing the blood to leak underneath the retina. This blood overlays the cones and rods, preventing them from capturing the light. As a consequence, black spots appear in the person's eyesight, progressively evolving towards blindness.

There is no curative treatment up to now for this disease [2]. A promising treatment is retinal vein cannulation (RVC) [3], [4], [5], it consists of introducing a needle inside the patient's eyeball to flush away the clot with a thrombolytic agent as shown in Fig. 1. Here, recently the first robotic sugery for RVC was performed in human patients [6]. The surgeon starts by a vitrectomy where he/she replaces the vitreous humour of the eye with $0.9 \%$ saline solution, namely a vitrectomy. The surgeon then performs a small incision on the sclera of the eyeball to position a trocar, creating a sealed access to the inside of the eye. The clinician slides the needle through the trocar to target the clotted vessel. Once punctured, the agent is injected to dissolve the

\footnotetext{
*This work was funded by a C3-fund (3E160419) from KULeuven

${ }^{1} \mathrm{KU}$ Leuven - University of Leuven, Department of Mechanical Engineering, 3001 Heverlee, Belgium laurent.schoevaerdts@kuleuven.be

${ }^{2}$ G. Borghesan, D. Reynaerts are members of Flanders Make, Leuven, Belgium.
}

clot. The whole procedure is performed with a stereoscopic microscope over the patient eye lens to guide the instrument towards the targeted site. Despite the modern visualization, depth perception remains poor, making that there is a risk that the surgeon pierces the clotted vessel and injects the agent underneath the retina, hence damaging the patient's eyesight.

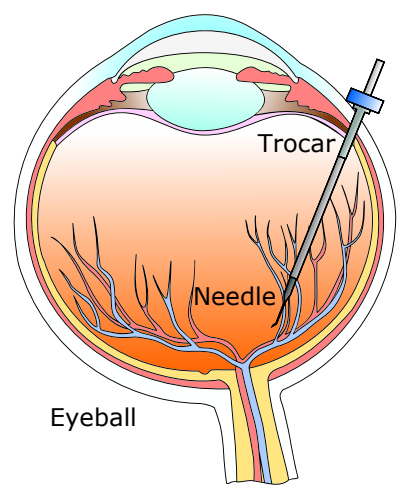

(a) Surgical instrument inserted inside the eyeball through a trocar.

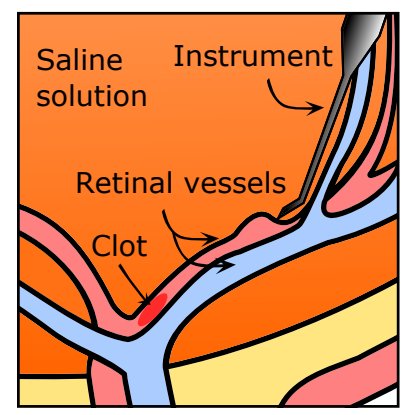

(b) Puncture of a retinal vessel in the vicinity of a clot.
Fig. 1: Cannulation of retinal vein with RVC dedicated needle.

\section{B. Proximity sensing for eye surgery}

Heretofore, research mainly investigated intraoperative Optical Coherence Tomography (iOCT) as a mean to provide intra-ocular proximity sensing. This technology provides 1D (A-scan), 2D (B-scan) or 3D (C-scan) iOCT images. The iOCT system shares the optical path of the stereoscopic microscope producing B- and C-scans. Alternatively, an optical fiber can be linked to the iOCT system to generate A-scans. With such a sensor, a distance to the retina can be estimated. Balicki et al. [7] report an OCT fiber integrated retinal pick in. Experiments on synthetic phantoms were conducted with the pick mounted on a robotic platform and linked to a custom developed iOCT device. Cheon et al. [8] and Borghesan et al. [9] developed algorithms using Ascans to detect the retinal surface on ex-vivo porcine and bovine eyes respectivel. Recently, Preceyes validated realtime distance measurement in clinical conditions with an OCT-probe [10]. While promising, OCT is expensive and not easy to integrate in miniature instruments.

In previous work, we used electrical bio-impedance sensing for puncture detection [11]. In this paper, we investigate the potential of bio-impedance sensing as an affordable 
alternative to iOCT for proximity sensing. Some prior work on electrical bio-impedance was conducted by Ray et al. for retinal stimulation with an electrode [12]. They report a technique using a platinum-iridium stimulation electrode to sense the proximity to the retina in living rats' eyes. They measure an impedance rise when approaching the surface. OCT B-scans are taken to estimate the actual distance to the retina when approaching. However no information is available beyond $500 \mu \mathrm{m}$. Majdi reports a similar rise in impedance when approaching the retina with a $1.6 \mathrm{~mm}$ outer diameter (OD) insulated probe over a range of $1 \mathrm{~mm}$ [13]. Majdi explains this impedance rise by a decreasing electrically excited neuronal surface, hence causing an increased access resistance of the retina.

This paper investigates further the relation between electrical bio-impedance and position of a probe relatively to the retinal vessels on ex-vivo enucleated pig eyes. We evaluate the suitability of such sensor for setting up virtual bounds to prevent probe contact with the vessel and whether the current technology allows consistent position assessment of the probe relatively to the vessel. We noticed that a transition point in the impedance magnitude indicates retinal proximity in a systematic manner. This pattern is independent of the magnitude and is therefore less dependent on environmental parameters. Finally a minimally-invasive measurement approach is presented to show how this technique could be developed in more realistic conditions.

\section{Procedure CONDitions And SET-UP}

\section{A. Workspace and environment inside the eyeball}

In RVO, the cannulation of the clotted vessel usually takes place in $0.9 \%$ saline solution as above mentioned. This means that the eyeball is filled with an electrically conductive liquid, namely an electrolyte. Besides, the surrounding organic tissue is also electrically conductive. Such properties allow a current to flow through. The measured electrical bioimpedance will depend mainly on the tissue properties, the distance into the saline solution and electrodes geometry. These aspects are discussed next.

\section{B. Set-up to measure the electrical bio-impedance and dis- tance}

In order to compute the relation between the impedance and the distance between the electrode tip and the retinal vessel, a dedicated set-up was developed. Fig. 2 shows the assembly for the experiment. The set-up is placed under a stereoscopic microscope. A stainless steel $150 \mu \mathrm{m}$ outer diameter electrode with a length of $10 \mathrm{~mm}$ is used as measurement means. The electrode is soldered in a 0.75 OD mm stainless steel tube, which is covered with a teflon tube to electrically insulate it. It is attached to a vertical linear micro-manipulator (M-423, Newport company, California), which is mounted on two other ones horizontally positioned. They are equipped with Vernier Micrometers on their side (SM-25, Newport company, California) so that 1 $\mu \mathrm{m}$ steps can be made. Below this stage, ex-vivo enucleated pig eyes are positioned on a frame such that the electrode can vertically approach the targeted vessel. The electrode is wired to a real-time impedance measurement device (Quadra, Eliko company, Estonia). This device measures impedance magnitude and phase at 15 different excitation frequencies at $1 \mathrm{kHz}$ sampling frequency, measuring impedance magnitudes from $1 k \Omega$ to $100 k \Omega$ with $0.5 \%$ accuracy. The data is acquired by USB. A distance sensor (Laser OADM12I6460 S35A, Baumer company, Switzerland) fixed to the vertical micro-manipulator measures the vertical displacement of the electrode. The sensor has a resolution of $2 \mu \mathrm{m}$ and measures at $100 \mathrm{~Hz}$. The laser position is acquired via Ethercat. Synchronous acquisition of both data allows computing the correlation between impedance magnitude/phase and the distance electrode tip to anatomy.

\section{BIO-IMPEDANCE CHARACTERIZATION WITH DISTANCE MEASUREMENT IN EYEGLOBE}

This section characterizes the bio-impedance measurements by comparing with laser distance measurements. First, the electrical model of the set-up is introduced. Second, typical impedance profiles are explained. Finally, a specific pattern that appears in the impedance measurement when approaching the retina is discussed.

\section{A. Electrical model of the measurement set-up in the eyeball}

The electrode is in contact with the saline solution while the second crocodile clip is clamped at the other side of the retina to the optic nerve as shown in Fig. 2. The electrical behavior is modelled by (1) [12]. The resistor $R_{s}$ represents the intrinsic resistive behavior of the saline solution and tissue. It also depends on the electrode geometry and contact surface. The capacitor $C_{s}$ models a phenomenon known as double layer capacitance. It originates from charges that accumulate at the probe interface. The excitation frequency of the applied voltage is $\omega$.

$$
Z_{\text {electrode }}=R_{s}+\frac{1}{j \omega C_{s}}
$$

It can be noticed that when working at high excitation frequencies, the parasitic capacitance effect greatly diminishes. The electrodes will thus mainly measure the resistive behavior of the electrolyte, which indicates the distance of the electrode tip to the retina. Further experiments use this principle.

\section{B. General Profile of the impedance vs the distance}

To measure the relation between impedance and distance, ex-vivo enucleated pig eyes are cut half open to access the retinal vessels. The vitreous humour is removed and replaced by $0.9 \%$ saline solution. The eye is then placed on a stand (see Fig. 2 set-up). A crocodile clip is attached to the optic nerve of the eye. Another crocodile clip is attached to the backside of the electrode. A voltage difference is applied between the outer shell of the eye and the instrument tip inside the eye. The electrical impedance will be a function of the distance between the electrode tip and the vessel. In order to determine the actual height of the vessel relatively 

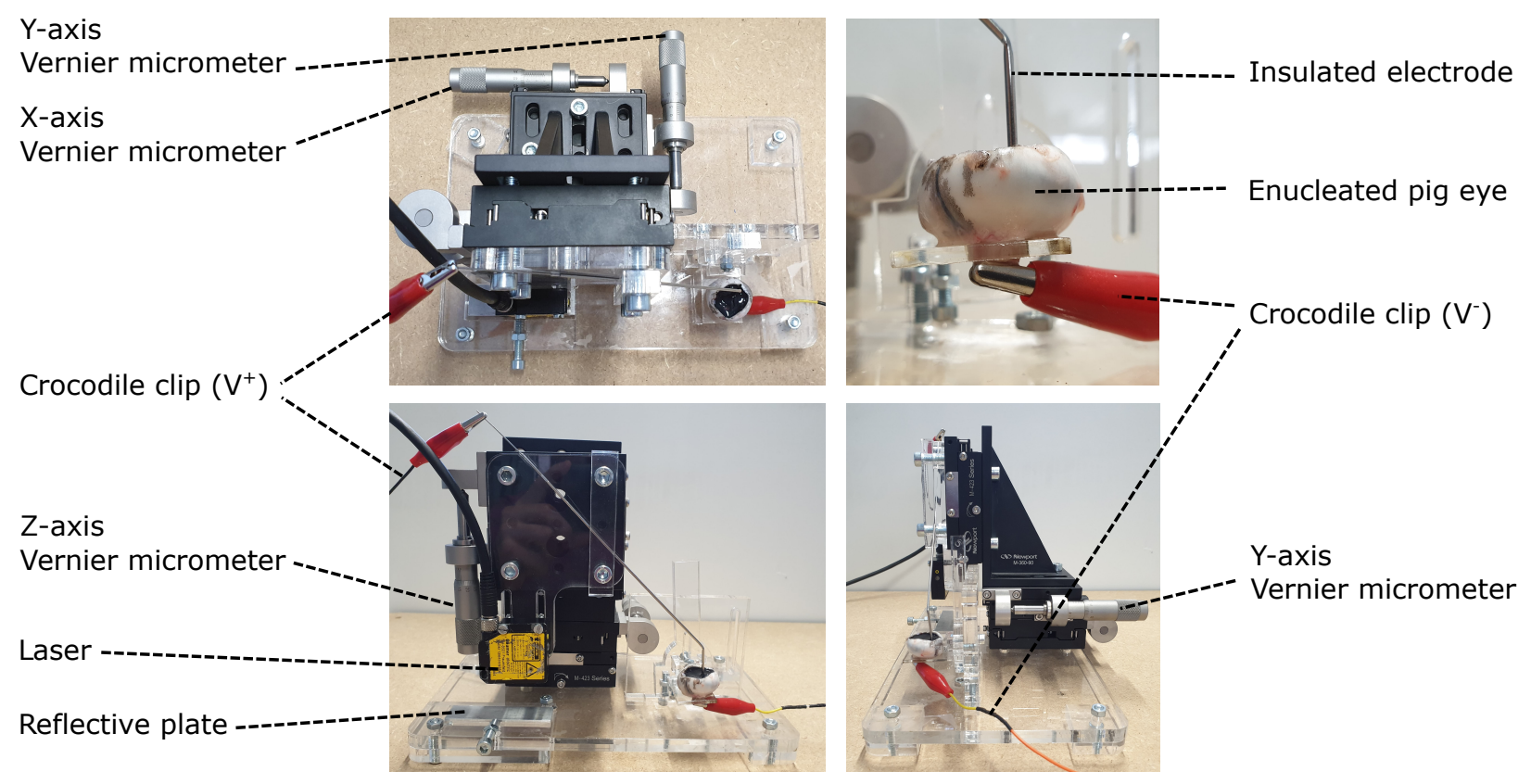

Fig. 2: Set-up to measure electrical bio-impedance on ex-vivo pig eyes. The insulated electrode is actuated vertically to approach the retina via a Z-axis micro-manipulator. An XY platform consisting of two other micro-manipulators align the electrode horizontally above the retinal structures. A laser mounted together with the stage of the insulated electrode measures the travel in Z-direction. A voltage difference is applied over the two crocodile clips.

to the set-up, first a contact is established with the targeted retinal vessel as shown in Fig. 3. This reference location is recorded by the laser. By touching the vessel slightly, the blood is pushed away and makes it turn white, informing that contact has been established with the electrode tip. The error on this registration step is estimated to be on average $100 \mu \mathrm{m}$ giving the quality of visual feedback.

Experiments with 5 pig eyes were conducted with the set-up in Fig. 2 to understand the general tendency of the impedance magnitude and phase when approaching the retina. The impedance was characterized over a range of 5 $\mathrm{mm}$ to the vessel.

The excitation frequency of the system was chosen to reduce the parasitic effect of the double layer capacitance. Fig. $4 \mathrm{a}$ and $4 \mathrm{~b}$ show the magnitude and phase over a roundtrip of $5 \mathrm{~mm}$ under the largest excitation frequency of the Quadra, namely $349 \mathrm{kHz}$. A transition region was observed in the magnitude in Fig. 4a in all the tests at a distance of $775 \mu m \pm 275 \mu m$.

\section{ACTIVE BOUNDARY PREVENTING RETINAL CONTACT}

This section explores the transitional phenomenon on impedance magnitude when getting closer to the retinal vessel. First, the phenomenon is explained. Then, an algorithm is derived to detect the transition. Finally, experiments are conducted to estimate the sensitivity and specificity of the proximity detection method.

\section{A. Transition detection}

Fig. 4a shows a specific pattern in impedance magnitude that may allow establishing an active boundary. This is in

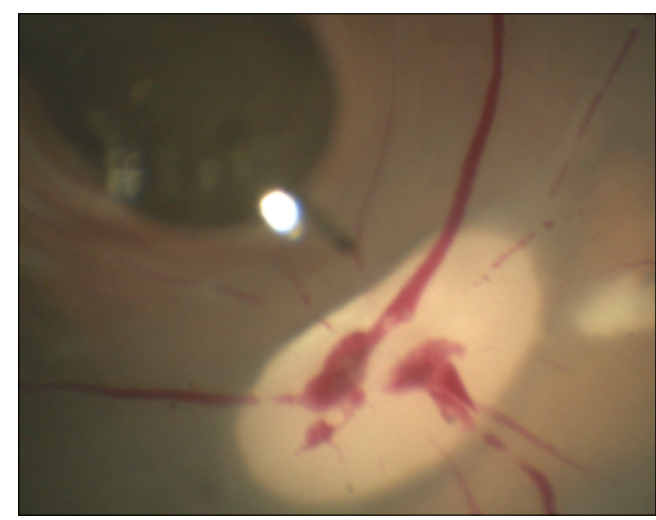

(a) Retinal vessels filled with blood before contact.

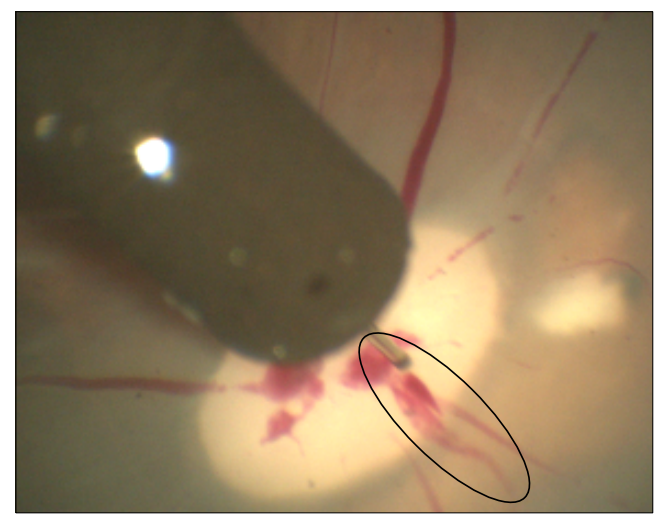

(b) The blood is seen to be flushed away when applying a slight pressure on the vessel.

Fig. 3: Procedure to assess contact between the electrode tip and retinal vessel to register the vessel. 


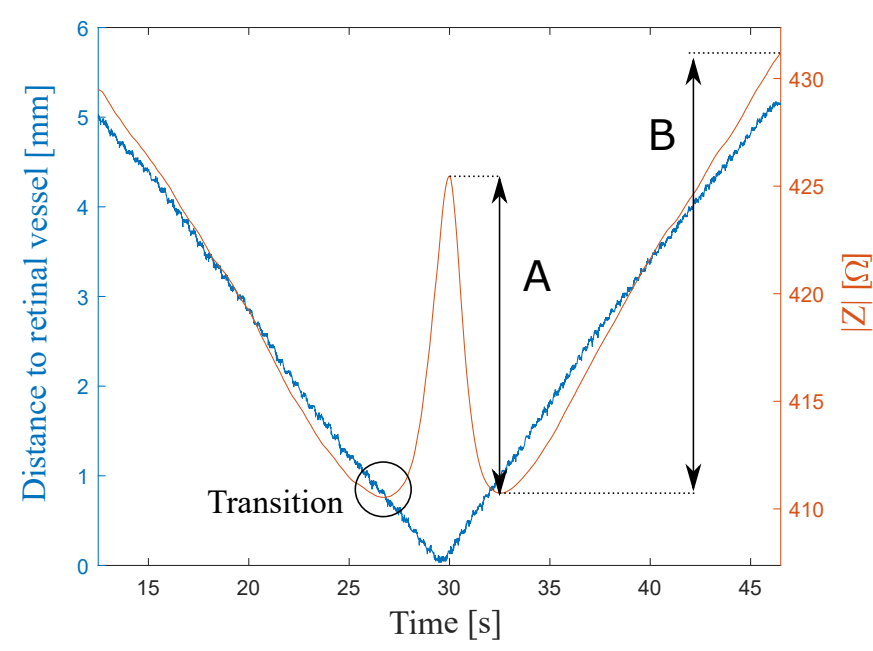

(a)

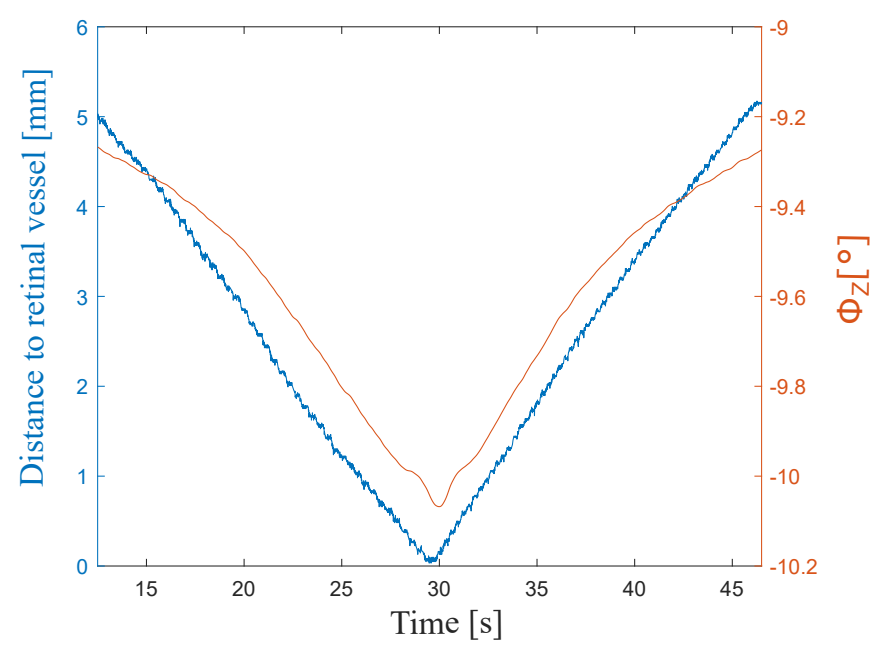

(b)

Fig. 4: Impedance magnitude (a) and phase (b) measured over time during one round-trip in an ex-vivo pig eye. The blue curve is the electrode - retina relative distance. Measurements run at $349 \mathrm{kHz}$. A and B refer to the parameters shown in Fig. 12.

contrast to the impedance magnitude which seems to depend on many parameters such as the eye condition. The pattern is common to all the measurements. Ray had observed a similar raise in impedance magnitude when approaching the retina [12] but did not report on the decrease of impedance when approaching at a larger distance. Neither was the transition detailed.

In order to detect this transition, a detection algorithm (Alg. 1) was designed that combines the impedance magnitude at $349 \mathrm{kHz}$ with the measured electrode speed. Indeed the transition is symmetric to the distance as shown on Fig. 4 but only when approaching the vessel too closely, one may want to issue a warning signal or stop command. That is why the motion direction of the electrode is used to differentiate when the practitioner is aiming at the vessel or retracting it, respectively when the speed is negative and positive. The algorithm 1 ran at $100 \mathrm{~Hz}$. The raw data noise from the laser sensor and the Quadra were filtered out.

\section{B. Experimental outcomes}

The detection was tested over 5 ex-vivo enucleated pig eyes prepared following the description in SubSec. III-B. Once that a retinal vessel was chosen, 10 round-trips were conducted at a distance of 4 or $5 \mathrm{~mm}$ away from the vessel. Hence 50 round-trips were achieved in total. Figure 5 displays typically variations of impedance magnitude and distance of four round-trips measured over time in one enucleated pig eye. A binary value overlays the graph when the algorithm 1 detects the transition region. The first constant region follows from calibration of $\sigma_{V e l}$ at the start of each cycle. The scalar $\alpha_{t}$ determines the lower and upper limits of the speed at steady state of the electrode, taking into account noise on the laser. If the speed is higher than $\left|\alpha_{t} * \sigma_{V e l}\right|$, the electrode is considered as moving. The constant was experimentally determined from one to be 0.08 and be kept constant for the 4 other pig eye experiments.

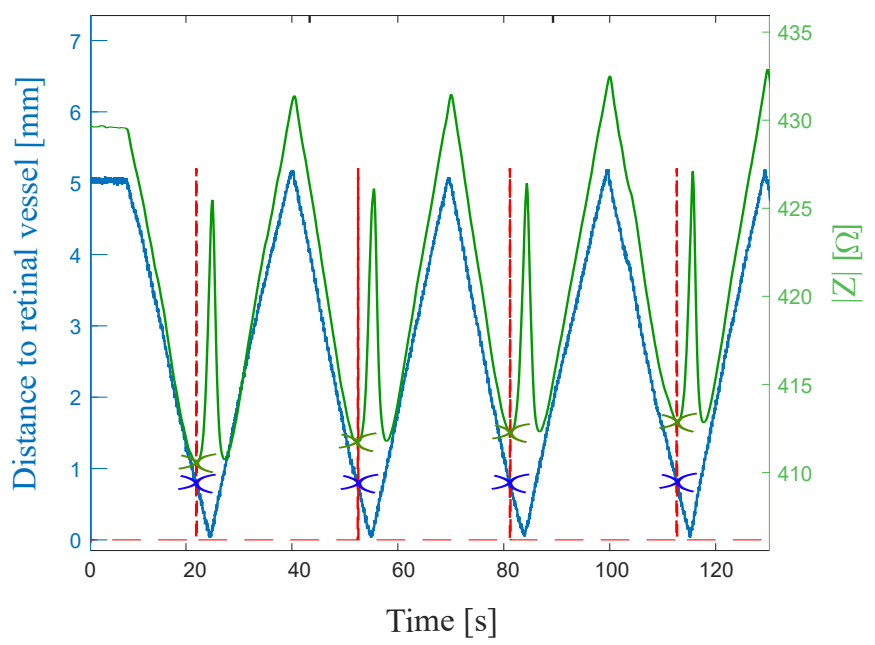

Fig. 5: Distance and impedance magnitude over 4 roundtrips nearby the retina. The red dashed curve indicates when a transition is detected by Alg. 1. The green and blue crosses respectively indicate the distance and magnitude at which the transitions are detected.

Over the 50 round-trips, 49 out of 50 transitions close to the vessels were detected. No transition was reported outside the expected range. The algorithm therefore had a sensitivity of $98 \%$ and specificity of $100 \%$. In the only undetected transition, noise on the position measurement was significantly higher than usual, causing poor speed measurements.

\section{Position assessment of the probe tip inside the eyeglobe}

Over the 50 round-trips, it was found that the transition takes place at a distance of $775 \mu \mathrm{m} \pm 275 \mu \mathrm{m}$. The measured magnitude peak to peak values over the 50 round-trips ranged 
Data:

$T_{i}$ : time at step i;

$d_{e l, i}$ : filtered distance measured by laser at step i;

$V_{e l, i}$ : electrode speed at step i;

$\sigma_{V e l}$ : standard deviation of noise over electrode speed

(derived from laser);

$|Z|_{i}:$ filtered impedance magnitude at step i;

$\delta|Z|_{i}$ : impedance magnitude derivative at step i;

$\alpha_{t}:$ threshold of $\sigma_{V e l}$ before transition detection;

\section{Result:}

Return 1 when transition is detected, otherwise 0;

\section{Intialize:}

$V_{e l, i}, \sigma_{V e l}$ and $|Z|_{0} \Rightarrow 0$

\section{Calibration during 10 seconds at rest with $\mathbf{N}=$ 10000 samples:}

compute mean $\mu_{Z n}=\frac{1}{N} \sum_{i=1}^{N}|Z|_{i}$ Compute

$\sigma_{V e l}=\frac{1}{N-1} \sum_{i=1}^{N}\left(|Z|_{i}-\mu_{Z n}\right)^{2}$

while experiment ongoing do

update $d_{e l, i},|Z|_{i}$

compute $V_{e l, i}=\frac{d_{e l, i}-d_{e l, i-1}}{T_{i}-T_{i-1}}$;

compute $\delta|Z|_{i}=\frac{|Z|_{i}-|Z|_{i-1}}{T_{i}-T_{i-1}}$

if

$\left(V_{e l, i}<=-\alpha_{t} \cdot \sigma_{V e l}\right) \&\left(\delta|Z|_{i-1}<0\right) \&\left(\delta|Z|_{i}>0\right)$

then

Transition detected $\Rightarrow$ return 1 ;

else

No transition detected $\Rightarrow$ return 0 ;

end

Algorithm 1: Method to detect the transition in impedance magnitude when approaching a retinal vessel.

from $[388 \Omega \pm 39 \Omega ; 410 \Omega \pm 38 \Omega]$. A drift was observed over all the experiments. Figures 5 and Fig. 6 show this drive for four round-trips in one experiment. Other experiments behave similarly. This is most likely due to some saline solution penetrating the buffer between the stainless steel probe and the teflon sleeve. The drift was found to be in the range of 3 to $30 \mathrm{~m} \Omega / \mathrm{s}$ over the experimental timespan. The total drift over each experiment was measured as 5\% to $66 \%$ of the peak to peak magnitude (ranging from 15 to $20 \Omega$ ) of a round-trip. The impedance phase ranged from $\left[-9.78^{\circ} \pm 0.5^{\circ} ;-9.25^{\circ} \pm 0.5^{\circ}\right]$. The most critical drift of the phase was $5 \cdot 10^{-4} o / s$. The total drift varied from $0 \%$ to 19 $\%$ of the peak to peak phase range for a round-trip (which corresponds to a range of 0.6 to $1.1^{\circ}$ ).

\section{Ex-vivo EXPERIMENTS ON A FULL PIG HEAD}

The previous experiments make use of the optic nerve to connect the crocodile clip. In clinical conditions, placing an electrode behind the eye would be highly invasive and undesirable. Here a first practical approach is proposed. We

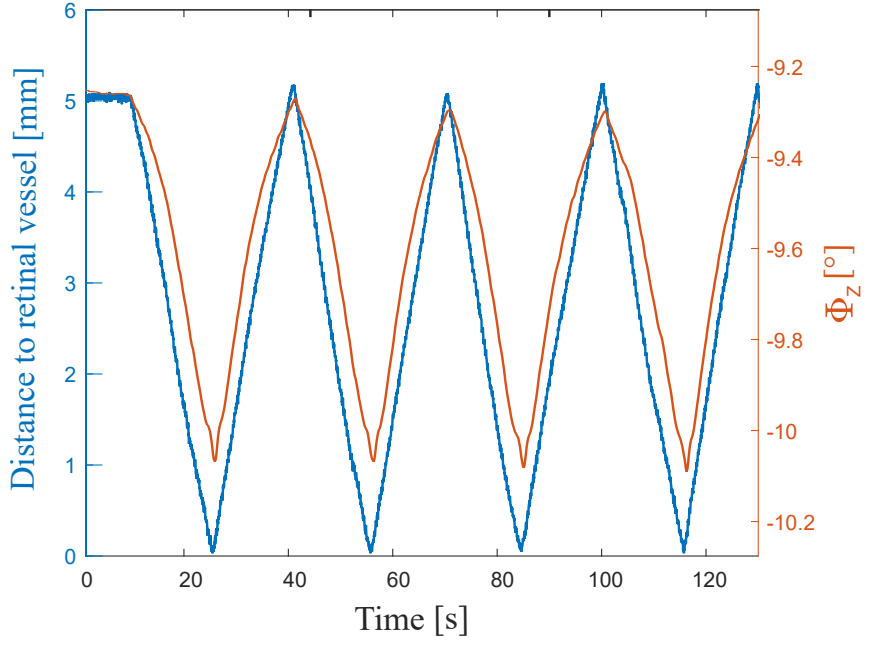

Fig. 6: Distance and impedance phase over 4 round-trips.

depart from noticing that the optic nerves of ones eyes are joint at the optic chiasm (see Fig. 7). Considering that these optic nerves are meant to transmit electrical signals based on external light stimuli, it follows that they are intrinsically electrically conductive. By connecting one electrode on each eye, a voltage difference should drive a current through both optic nerves. As far as the authors are aware of, this is the first time such route is followed for proximity sensing. This measurement principle gives a minimally-invasive access to the optic nerve of the operated eye. In this work, a pig head is used to show the measurement principle. A similar set-up to Fig. 2 is used with a straight insulated electrode that was aligned nearby the operated eye. A sclerotomy is made to introduce the electrode inside the eyeglobe. The electrode is connected to $V^{+}$. Another sclerotomy allows a $2 \mathrm{~mm}$ OD endoscope (Karl Storz, Miniature - endoscope, $0^{\circ}$, semirigid) to fit inside the eye to light up the scene and give visual feedback. Latter endoscope is connected to a camera (Allied Vision, Prosilica GC) to record the operation. Another $2 \mathrm{~mm}$ OD electrode, under $V^{-}$voltage, is positioned against the left eye and kept in place by closing the eyelid. Figures 7 and 8 show the overall layout.

Manipulating an ex-vivo pig head is highly cumbersome. As a result, a retinal detachment took place while this is to be avoided. In reality, here the experiment was continued to provide some first relevant data. The straight insulated electrode was moved three times forward and backward towards the retinal detachment while recording the electrical impedance and the endoscope images as shown on Fig. 9. During the experiments, the distance to the detached retina was estimated from the camera images. Figure 10 displays the impedance magnitude measured throughout the experiment. Contact with the retina was determined when the retinal detachment was pushed away by the electrode. This explains why the distance displayed on Fig. 10 goes slightly below $0 \mathrm{~mm}$.

Figure 12 displays a typical impedance magnitude pattern measured during the experiment. "A" and "B" are respec- 


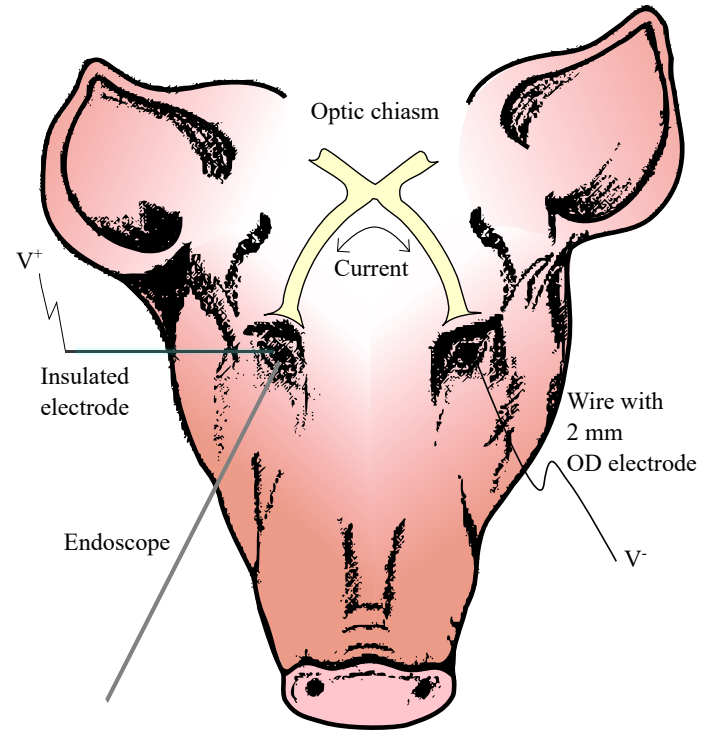

Fig. 7: Schema of the measurement approach making use of the optic chiasm to measure proximity.
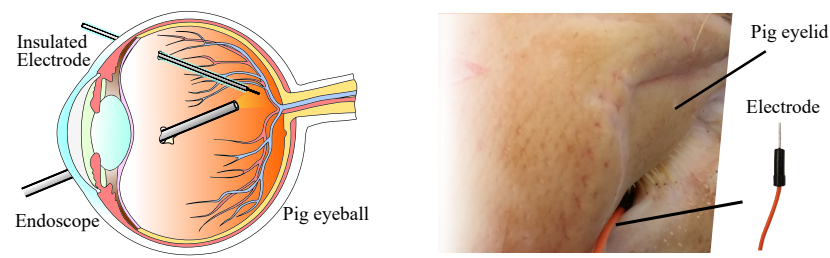

Fig. 8: Left - schema of the operated right pig eye. The insulated probe is moved towards the retina. The endoscope records the motion. Right - the electrode is brought into contact with the sclera of the left eye and maintained in this position by closing the eyelid.

tively the amplitudes of the peak beyond the transition and the amplitude rise outside the transition zone as shown in Fig. 12. Several differences are observed compared to the enucleated pig eyes. First, the peak amplitude "A" upon approaching the retina is more pronounced with the pig head with "A" equal to $25 \Omega$ in Fig. 10 versus $10 \Omega$ on ex-vivo pig eyes from Fig. 5. This phenomenon may find its origin in the new electrical path involving the optic nerve and the two eyes. Moreover, the retinal detachment may also alter the amplitude of the peak. Second, the measured amplitude "B" only rises up to $2 \Omega$ versus $20 \Omega$ for the enucleated eyes. The retinal detachment may explain this as upon approach, the retinal surface surrounds the electrode tip, reducing the impact of the tip/retina distance over the impedance magnitude. Due to the limited field of view of the scope, the range is limited to $3 \mathrm{~mm}$. Measuring over a larger range would have most likely underlined further the transition point nearby the retina. Last, the transitions are visible in Fig. 10 at respectively $2.1,1.75$ and $1.6 \mathrm{~mm}$ from the surface. The new electrical path and retinal detachment may have altered the outcome in comparison with the experiments on ex-vivo pig eyes.
In contrast, the impedance phase from Fig. 11 shows a similar behavior and amplitude as Fig. 6. The drift seems less pronounced but is due to the difference in time scale between the two experiments. The experiment on ex-vivo pig eyes lasted about 5 times longer than the experiment on the $e x$ vivo pig head. One can also notice that the phase is slightly more negative for the ex-vivo pig head, meaning a more capacitive impedance. This observation may be explained by the difference in electrical paths and electrodes between the two experiments. Indeed, the experiment on pig eyes uses a crocodile clip on the optic nerve while a simple electrode is used on the left eye of the pig head.

This experiment was mainly meant to show the feasibility of such measurement in a minimally invasive way and the measurement of the transition pattern in a more realistic case. We could confirm the presence of the transition region also in this experiment. Although there is some variation compared to the ex-vivo model (confirming the importance of realistic models) the results are promising and urging further exploration also in in-vivo models where the behaviour might still alter.

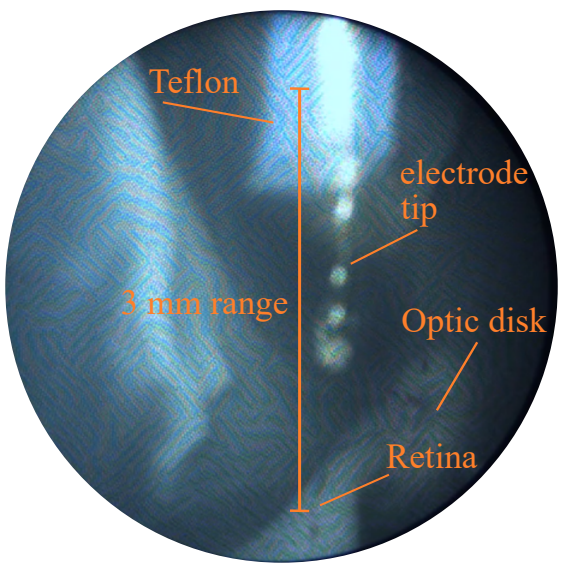

Fig. 9: Electrode executing round-trips between start and end point. The retina is visibly detached. The endoscope shows a range of $3 \mathrm{~mm}$ over which the electrode tip is displayed.

\section{DISCUSSION AND FUTURE PERSPECTIVES}

The transitions in impedance magnitude showed up for all experiments. This measure can thus be used to assess proximity of the retinal vessel. On Fig. 5 and Fig. 10, one can observe a drift over time. This makes that the absolute amplitude can at this stage not be used to estimate the distance itself. To tackle this effect in further experiments, we plan to use noble metal electrodes to remove possible galvanic currents and and make sure the saline solution does not penetrate the buffer between the teflon tube and stainless steel probe. Overall, the impedance magnitude is strongly dependent on the contact surface of the electrode and the crocodile clip.

Furthermore, the impedance phase was also shown to be strongly correlated to the distance between the electrode tip and the retina as shown in Fig. 6 and Fig. 11. The impedance 


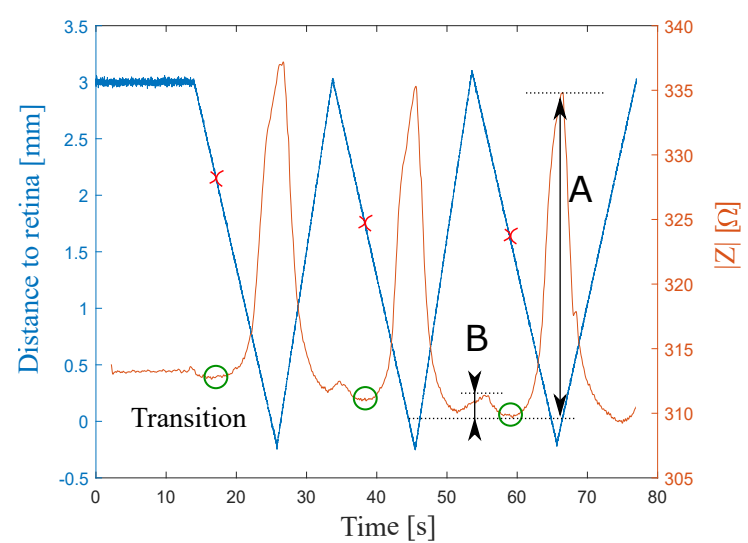

Fig. 10: Distance and impedance magnitude measured over time during three round-trips in an ex-vivo pig head. The "0 mm" reference corresponds to contact with the retinal detachment. The red crosses mark the distance at which a transition occurs (Green circles). A and B refer to the parameters shown in Fig. 12.

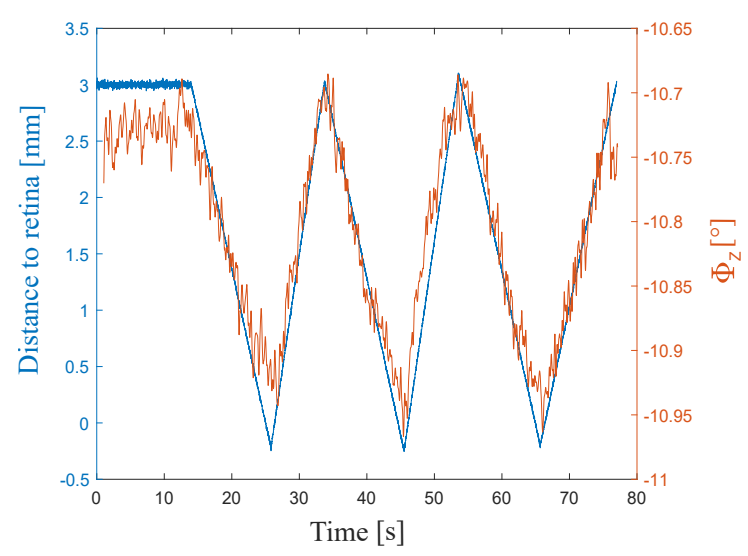

Fig. 11: Distance and impedance phase measured over time during three round-trips with the insulated electrode in the eye from an ex-vivo pig head. The distance "0 mm" represents a contact with the retinal detachment).

phase seems less sensitive to drift. At high excitation frequency, all parasitic capacitance effects are significantly reduced. As a matter of fact, Eq. 1 suggests that the phase tends to 0 as the pulsation $\omega$ rises significantly. One can notice that the phase becomes more capacitive (negative) when approaching the retinal vessel. As the electrode approaches the vessel, the resistance decreases while the capacitance stays almost unaffected. From 1, the phase can be computed as

$$
\Phi_{Z_{\text {electrode }}}=\operatorname{atan}\left(\frac{-1}{\omega R_{S} C_{S}}\right)\left[^{o}\right]
$$

confirming that the phase decreases as $R_{S}$ decreases. Finally, the experiment on the pig head showed that it is also possible to detect a similar pattern in a more realistic set-up. Future work will investigate safety aspects, e.g. current related risks or heating of the anatomical structures.

In future work, we plan to investigate how to modify a needle previously developed for puncture detection by electrical bio-

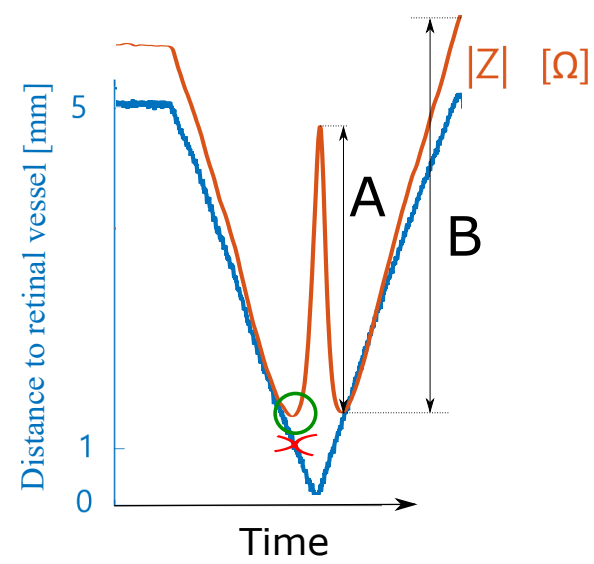

Fig. 12: Typical profile of impedance magnitude when the electrode approaches the retinal layer at " $0 \mathrm{~mm}$ ". The red cross marks the distance at which a transition occurs (green circle). A is the peak upon contact with the retina. B is the impedance rise upon complete recession of the electrode over the range of motion.

impedance measurement [11] to sense vessel proximity while cannulating. In a robotic system, proximity measure could be used to automatically increase damping when crossing the boundary. A second relevant use case would offer the practitioner an auditory cue upon crossing of the transition. The surgeon could then judge whether to adjust the robot damping him/herself. People can react to an audio feedback within $200 \mathrm{~ms}$. Nominal instrument speeds can be considered at $500 \mu \mathrm{m} / \mathrm{s}$ [14]. This means the practitioner could stop the needle within $100 \mu \mathrm{m}$. This is reasonable considering that the transition happens at $775 \mu \mathrm{m} \pm 275 \mu \mathrm{m}$ from the retinal vessel. In addition, such approach might also be beneficial in retinal membrane peeling. The surgeon has to approach the retina, grab the retinal layer and peel it off by circling the instrument within the workspace of the eyeball. Proximity sensing could be an easy and affordable way to set up a virtual safety bound here.

\section{CONCLUSION}

This work reported a novel way and affordable to sense retinal vessel proximity by using electrical bio-impedance sensor. A set-up with a $150 \mu m$ outer diameter electrode was developed to measure the impedance between a pig eye sclera and the electrode tip inside the eye. The setup was tested on 5 ex-vivo enucleated pig eyes, measuring impedance phase/magnitude and distance between the electrode tip and the retinal vessel. All the experiments underlined a repetitive pattern in impedance magnitude and a correlation between impedance phase and electrode tipretina distance. An algorithm allowed to automatically detect this pattern at a distance of $775 \mu \mathrm{m} \pm 275 \mu \mathrm{m}$ of the retinal vessels with a sensitivity of $98 \%$ and a specificity of $100 \%$ over the 50 trials on enucleated pig eyes. A method to apply the technology in a more realistic setting making use of the optic chiasm was devised. Experiments on an ex-vivo pig 
head showed that the phenomenon - although less outspoken - is still present. Further validation is needed to study this response in greater detail. However, this already opens the door to an affordable way to measure the retinal proximity in eye surgery where depth perception remains critical.

\section{REFERENCES}

[1] S. R. et al., "The prevalence of retinal vein occlusion: pooled data from population studies from the united states, europe, asia, and australia." Ophthalmology, vol. 140, no. 2, pp. 313-319, 2010.

[2] W. M. Tang and D. P. Han, "A study of surgical approaches to retinal vascular occlusions." Archives of Ophthalmology, vol. 118, no. 1, pp. 138-143, 2000.

[3] JN Weiss and LA Bynoe. Injection of tissue plasminogen activator into a branch retinal vein in eyes with central retinal vein occlusion. Ophthalmology, vol. 108, no. 12, pp. 2249-2257, 2001.

[4] T. Nakano et al., "A parallel robot to assist vitreoretinal surgery", International Journal of Computer Assisted Radiology and Surgery, vol. 4, no. 6, pp. 517-526, 2009.

[5] I. Fleming, M. Balicki, J. Koo, I. Iordachita, B. Mitchell, J. Handa, G. Hager and R. Taylor. "Cooperative Robot Assistant for Retinal Microsurgery", Medical Image Computing and Computer-Assisted Intervention (MICCAI), vol. 5242, pp. 543-550, 2008.

[6] A. Gijbels, J. Smits, L. Schoevaerdts, K. Willekens, E. B. Vander Poorten, P. Stalmans, and R. D., "In-human robot-assisted retinal vein cannulation, a world first." Annals of Biomedical Engineering, pp. 1-10, May, 2018.

[7] M. Balicki, J.-H. Han, I. Iordachita, P. Gehlbach, J. Handa, R. Taylor, and J. Kang, "Single fiber optical coherence tomography microsurgical instruments for computer and robot-assisted retinal surgery," in Medical Image Computing and Computer-Assisted Intervention-MICCAI 2009. Springer, 2009, pp. 108-115.

[8] G. W. Cheon, Y. Huang, J. Cha, P. L. Gehlbach, and J. U. Kang, "Accurate real-time depth control for cp-ssoct distal sensor based handheld microsurgery tools." Biomedical Optics Express, vol. 6, no. 5, pp. 1942-1953, 2015.

[9] G. Borghesan, D. Bouget, E. Lankenau, R. Neffin, P. Koch, K. Willekens, P. Stalmans, D. Reynaerts, and E. Vander Poorten, "Single fiber oct-based retina detection for robot-assisted retinal vein cannulation," in 6th joint workshop on New Technologies for Computer/Robot Assisted Surgery 2016.

[10] https://www.linkedin.com/pulse/preceyes-validates-real-time-distancemeasurement-retinal-gerrit-naus

[11] L. Schoevaerdts, L. Esteveny, A. Gijbels, J. Smits, D. Reynaerts, and E. Vander Poorten, "Design and evaluation of a new bioelectrical impedance sensor for micro-surgery: application to retinal vein cannulation.'International Journal of Computer Assisted Radiology and Surgery (IJCARS), pp. 1-10, August, 2018.

[12] A. Ray, L. Lai-hang Chan, A. Gonzalez, M. S. Humayun, and J. D. Weiland, "Impedance as a method to sense proximity at the electroderetina interface." Transactions on Neural Systems and Rehabilitation Engineering, vol. 19, no. 6, pp. 696 - 699, 2011.

[13] J. A. Majdi, S. Minnikanti, N. Peixoto, A. Agrawal1, and E. D. Cohen, "Access resistance of stimulation electrodes as a function of electrode proximity to the retina." J. Neural Eng., vol. 12, no. 1, 2015.

[14] J. Smits, M. Ourak, A. Gijbels, L. Esteveny, G. Borghesan, L. Schoevaerdts, K. Willekens, P. Stalmans, E. Lankenau, H. SchulzHildebrandt, G. Hüttmann, D. Reynaerts, and E. Vander Poorten, "Development and experimental validation of a combined fbg force and oct distance sensing needle for robot-assisted retinal vein cannulation." International Conference on Robotics and Automation (ICRA), May, 2018. 